\title{
A New Framework for Measuring Volume of Axisymmetric Food Products using Computer Vision System Based on Cubic Spline Interpolation
}

\author{
Joko Siswantoro, Endah Asmawati \\ Department of Informatics Engineering, Faculty of Engineering, Universitas Surabaya \\ Jl. Kali Rungkut, Surabaya, 60293, Indonesia \\ joko_siswantoro@staff.ubaya.ac.id,endah@staff.ubaya.ac.id
}

\begin{abstract}
Volume is an important factor to determine the external quality of a food product. The volume measurement of food product is not a simple process if it is performed manually. For alternative, several volume measurement methods for food products have been proposed using 2D and 3D computer vision. Disk method and frustum cone method have been applied in many 2D computer visions to approximate the volume of axisymmetric food products. These methods were less in accuracy, since it used piecewise linear function to approximate the boundary of the object. This paper aims to propose a new framework for measuring the volume of axisymmetric food product based on cubic spline interpolation. Cubic spline interpolation is employed to construct a piecewise continuous polynomial of the boundary of object from captured image. The polynomial is then integrated to approximate the volume of the object. The simulation result shows that the proposed framework produced accurate volume measurement result.
\end{abstract}

Keywords-volume; axisymmetric; food products; computer vision; cubic spline interpolation

\section{INTRODUCTION}

There are several applications of volume measurement in production and processing a food product, such as for grading, sorting, and determining other physical food properties [1]. Water displacement method based on Archimedes' principle is a conventional way for measuring the volume of a food product. This method is less in accuracy, time consuming, and can damage the measured object [2]. An alternative approach for measuring the volume of food products is using computer vision system (CVS). By using CVS for volume measurement, the accuracy can be increased; the required time can be decreased, as well as food product failure can be minimized. Several CVSs have been developed for measuring the volume of food products either using 2D or 3D CVS [3].

Almost all 2D CVS for volume measurement assumed that measured object has axisymmetric shape [2, 4-7]. An object has axisymmetric shape if its cross-sections that are perpendicular to its rotation axis is circular shape, such as watermelon, egg, orange, lemon, lime, and ellipsoidal ham
[8]. By using this assumption, the volume of object can be obtained by volume of solid of revolution method in Calculus [9]. In measuring the volume of food products, 2D CVS extracted the cross section of object that is parallel to rotation axis from captured image.

Sabliov, et al. [10], Du and Sun [5], and Wang and Nguang [2] have proposed 2D CVS to measure volume of axisymmetric food products using frustum cone method. They assumed that the boundary curve of object cross section that is parallel to rotation axis as piecewise continuous line. Bridge, et al. [4] and Koc [6] have proposed 2D CVS 2D CVS using disc method to measure volume of egg and watermelon, respectively. They assumed that the boundary curve of object-cross section that is parallel to rotation axis as pieces of horizontal line. The results of these approaches have a low accuracy, because a linier function has been used to approximate the boundary of object crosssection which is generally a non-linear function. Furthermore, CVSs proposed by Sabliov, et al. [10], Bridge, et al. [4], and Koc [6] were not fully automatic. They used different software for image processing and volume calculation.

In volume measurement using 3D CVS, several images of object were captured from different views [1, 11-15]. The images were then processed to reconstruct the surface of object or to generate $3 \mathrm{D}$ random point inside the object. In general, 3D CVS does not need any assumption about the shape of object. Therefore, it can be used to measure both axisymmetric object and irregularly shaped objects. Although, most of proposed 3D CVSs for volume measurement produced volume in high accuracy, the computational cost and computational time of the systems were very high, due to large number of images should be acquired and processed.

One of strategy to increase the accuracy of volume measurement of axisymmetric food product using volume of solid of revolution is by choosing an appropriate function to represent the boundary curve of object-cross section. Cubic spline interpolation is a numerical method for function approximation by using third order piecewise-polynomial 